
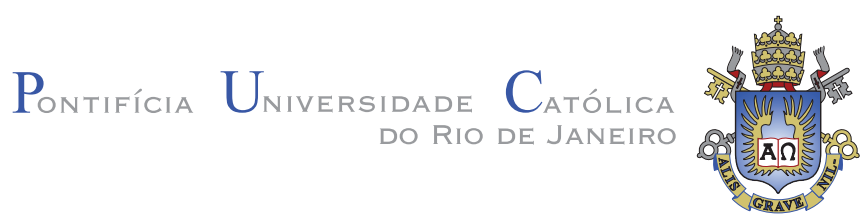

Ygor Hecht Speranza

\title{
Animação de fratura de objetos baseada em agrupamentos de vértices
}

\section{Dissertação de Mestrado}

Dissertação apresentada como requisito parcial para obtenção do grau de Mestre pelo Programa de Pós-graduação em Informática do Departamento de Informática da PUC-Rio

Orientador: Prof. Waldemar Celes Filho 

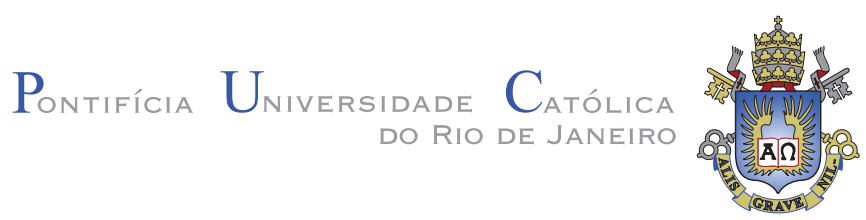

Ygor Hecht Speranza

\section{Animação de fratura de objetos baseada em agrupamentos de vértices}

Dissertação apresentada como requisito parcial para obtenção do grau de Mestre pelo Programa de Pós-graduação em Informática do Departamento de Informática do Centro Técnico Científico da PUC-Rio. Aprovada pela comissão examinadora abaixo assinada.

Prof. Waldemar Celes Filho Orientador Departamento de Informática - PUC-Rio

Prof. Bruno Feijó Departamento de Informática - PUC-Rio

Prof. Ivan Fabio Mota de Menezes Engenharia Mecânica - PUC-Rio

Prof. Helio Côrtes Vieira Lopes Departamento de Informática - PUC-Rio

Prof. José Eugênio Leal Coordenador Setorial do Centro Técnico Científico - PUC-Rio 
Todos os direitos reservados. Proibida a reprodução total ou parcial do trabalho sem autorização da universidade, do autor e do orientador.

\section{Ygor Hecht Speranza}

Graduou-se em Matemática Aplicada na Universidade Federal do Rio de Janeiro (UFRJ), com ênfase em computação. Realizou pesquisa em bioinformática através da Fundação Osvaldo Cruz (Fiocruz) e trabalhou junto a empresa K2 Sistemas no desenvolvimento de software de processamento de imagens e georeferenciamento. Desenvolveu junto com os seus orientadores durante o Mestrado técnica para animação de objetos fraturáveis.

Ficha Catalográfica

Speranza, Ygor

Animação de fratura de objetos baseada em agrupamentos de vértices / Ygor Hecht Speranza; orientador: Waldemar Celes Filho. - Rio de Janeiro : PUC-Rio, Departamento de Informática, 2012.

v., 74 f: il. ; $29,7 \mathrm{~cm}$

1. Dissertação (Mestrado em Informática) - Pontifícia Universidade Católica do Rio de Janeiro, Departamento de Informática.

Inclui referências bibliográficas.

1. Informática - Tese. 2. Animação de Objetos. 3. Modelo de Fratura. 4. Modelo de Deformação. 5. Segmentação de Malhas. 6. Dinâmica de Corpos Deformáveis. I. Celes Filho, Waldemar. II. Pontifícia Universidade Católica do Rio de Janeiro. Departamento de Informática. III. Título. 


\section{Agradecimentos}

Ao meu orientador Waldemar Celes, por todo o empenho e dedicação como professor e incentivador.

Ao CNPq e à PUC-Rio, pelos auxílios concedidos, sem os quais este trabalho não poderia ter sido realizado.

A todos os meus familiares, com agradecimentos especiais à Nayara e Ana, imensamente fundamentais para esse trabalho de muitas formas.

Aos amigos Paulo e Peter pela incansável ajuda.

Aos meus colegas da PUC-Rio, ao lado dos quais essa jornada tornou-se menos árdua. 


\section{Resumo}

Speranza, Ygor; Celes Filho, Waldemar. Animação de fratura de objetos baseada em agrupamentos de vértices. Rio de Janeiro, 2012. 74p. Dissertação de Mestrado — Departamento de Informática, Pontifícia Universidade Católica do Rio de Janeiro.

Neste trabalho, estendemos o método de simulação de objetos deformáveis de Muller et al. (2005) para simular fraturas. Em Muller et al., os vértices da superfície do objeto são tratados como partículas, sujeitas a forças externas ao objeto e a uma força interna de restituição, que tenta restaurar a forma do objeto através de uma técnica de casamento de forma. Esse método permite-nos simular efeitos como alongamento e flexão de forma estável e, por ser geometricamente motivado, é ideal para situações que não exijam realismo físico, como a área de animação e jogos. Muller et al. propõem uma forma de simulação composta, onde objetos deformáveis podem funcionar como uma composição de agrupamentos de vértices de sua superfície: esses agrupamentos agem como objetos deformáveis em si. Nossas contribuições concentram-se na variação deste modelo. Propomos utilizar o método de Attene et al. (2006) de segmentação hierárquica de superfícies para determinar de forma automática agrupamentos que sejam partes naturais do objeto. Criamos também uma técnica para determinar de forma suave a influência dos agrupamentos em cada vértice, levando em consideração aspectos globais e locais do objeto. Por fim, estabelecemos o algoritmo para a detecção de fraturas entre os agrupamentos e a execução da ruptura correspondente. Utilizamos um conjunto de objetos para provar que nosso método é capaz de simular fraturas naturalmente, podendo ser usado tanto em sistemas os quais exijam resultado mais simples, contudo em tempo real, quanto em sistemas que necessitam de fraturas apresentando maior riqueza de detalhes.

\section{Palavras-chave}

Animação de Objetos; Modelo de Fratura; Modelo de Deformação ; Segmentação de Malhas; Dinâmica de Corpos Deformáveis. 


\section{Abstract}

Speranza, Ygor; Celes Filho, Waldemar (advisor). Object Fracture Animation based on Vertex Clusters. Rio de Janeiro, 2012. 74p. MSc. Dissertation — Departamento de Informática, Pontifícia Universidade Católica do Rio de Janeiro.

In this work, we extend the simulation method for deformable objects proposed by Muller et al. (2005) so as to be able to simulate fracturing. In Muller et al., the object surface vertices are handled such as particles in a particle system: moved by external forces and by internal restitution forces, which try to restore the original object form using a shape matching technique. This method allow us to simulate stretching and twisting effects in a stable manner, and, because it is geometrically motivated, it is ideal to situations which does not demand physical realism, as in games and animation. Muller et al. propose in their work a composed simulation mode, in which deformable objects work like a composition of clusters of its surface vertices: these clusters behave like deformable objects on their own. Our contributions focus on a variation of this model. We propose the hierarchical vertex surface segmentation method from Attene et al. (2006) as an automatic way of determining clusters which are natural parts of the object. We also created a technique to smoothly calculate the influence of the clusters in each vertex, considering global and local aspects of the object. Finally, we established an algorithm which detects fractures between vertex clusters and which breaks the object surface accordingly. We employed a set of $3 \mathrm{D}$ objects to demonstrate that our method is capable of naturally simulating fractures, for systems which demand simpler, real-time results, as well as for systems which need richer and more detailed fractures.

\section{Keywords}

Object Animation; Fracture Model; Deformation Model; Mesh Segmentation; Soft Body Dynamics. 


\section{Sumário}

1 Introdução 11

1.1 Introdução 11

$\begin{array}{lll}1.2 & \text { Trabalhos Relacionados } & 14\end{array}$

2 Segmentação e Deformação $\quad 16$

2.1 Visão Geral 16

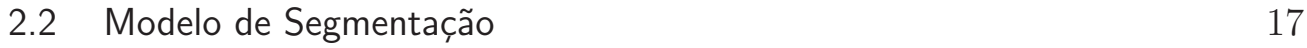

2.3 Heurísticas para Segmentação 28

2.4 Modelo de Deformação 29

3 Fratura $\quad 39$

3.1 Detecção de Separação de Agrupamentos 39

$\begin{array}{ll}3.2 & \text { Algoritmo de Fratura }\end{array}$

4 Resultados $\quad 44$

4.1 Simulações 44

4.2 Desempenho 56

5 Conclusão e Trabalhos Futuros $\quad 60$

6 Referências Bibliográficas $\quad 63$

A Apêndice I-Implementação $\quad 65$

$\begin{array}{lll}\text { A.1 Linguagem } & 65\end{array}$

A.2 Visualização $\quad 65$

A.3 Código 65

A.4 Estrutura de Classes $\quad 66$

$\begin{array}{lll}\text { A.5 Matrizes } & 69\end{array}$

B Apêndice II - Estabilidade de Sistemas Massa-Mola $\quad 73$

B.1 Sistema Massa-Mola Clássico 73

B.2 Variação do Sistema Massa-Mola 74 


\section{Lista de figuras}

2.1 Contração entre dois agrupamentos 18

2.2 Contrações entre agrupamentos e grafo de conexões 19

2.3 Pontos sendo aproximados por um plano 21

2.4 Determinação dos parâmetros $\mathbf{e}_{\mathbf{0}}$ e $r$ do cilindro 24

2.5 Influência local dos agrupamentos em vértice. 27

2.6 Diagrama de grafo de conexões entre agrupamentos 29

2.7 Laço principal do modelo de deformação 30

2.8 Modos de deformação 31

3.1 Fratura entre dois agrupamentos 40

3.2 Duplicação de vértice 42

4.1 Simulação de 5 objetos baseados no modelo "Bola de Plástico" 46

4.2 Diferentes padrões de fratura 46

4.3 "Bola de Plástico" sendo pressionado 47

4.4 Semgentação dos modelos "Jarro" e "Vaso" 48

4.5 Comparação do uso das primitivas na segmentação 49

4.6 Simulação do modelo "Jarro" 50

4.7 Diferentes padrões de fratura para "Vaso" simples e detalhado 51

4.8 Segmentação do modelo "Bexiga" 51

4.9 Alta tolerância do modelo "Bexiga" a deformações 52

4.10 "Bexiga" estourando 53

4.11 Detalhe do estouro do objeto "Bexiga" 54

4.12 Modelo "Janela"

4.13 Segmentação do modelo "Janela"

4.14 Simulação do objeto "Janela" 56

4.15 Objeto "Janela" configurado de forma a reagir como se fosse um objeto elástico 57

4.16 Gráfico do desempenho da simulação $\quad 59$

$\begin{array}{lll}\text { A.1 Diagrama de classes } & 67\end{array}$

A.2 Diagrama de classes do pacote de matrizes 70 


\section{Lista de tabelas}

4.1 Tempo de execução médio das duas fases do pré-processamento dos objetos simulados, em segundos.

4.2 Percentual do tempo de pré-processamento o qual decorre do algoritmo de segmentação

4.3 Desempenho em FPS obtida a partir das simulações executadas 
But we must not follow those who advise us, being men, to think of human things, and being mortal, of mortal things, but must, so far as we can, make ourselves immortal, and strain every nerve to live in accordance with the best thing in us; for even if it be small in bulk, much more does it in power and worth surpass everything.

Bertrand Russel, History of Western Philosophy. 